\title{
Spatiotemporal Activation of Lumbosacral Motoneurons in the Locomotor Step Cycle
}

\author{
SERGIY YAKOVENKO,${ }^{1}$ VIVIAN MUSHAHWAR, ${ }^{1}$ VERONIQUE VANDERHORST, ${ }^{2}$ GERT HOLSTEGE, ${ }^{2}$ \\ AND ARTHUR PROCHAZKA ${ }^{1}$ \\ ${ }^{1}$ Center for Neuroscience, University of Alberta, Edmonton, Alberta T6G 2S2, Canada; and ${ }^{2}$ Department of Anatomy, \\ University of Groningen, 9713 AV Groningen, The Netherlands
}

Received 11 June 2001; accepted in final form 6 November 2001

Yakovenko, Sergiy, Vivian Mushahwar, Veronique VanderHorst, Gert Holstege, and Arthur Prochazka. Spatiotemporal activation of lumbosacral motoneurons in the locomotor step cycle. J Neurophysiol 87: $1542-1553,2002 ; 10.1152 / j n .00479 .2001$. The aim of this study was to produce a dynamic model of the spatiotemporal activation of ensembles of alpha motoneurons (MNs) in the cat lumbosacral spinal cord during the locomotor step cycle. The coordinates of MNs of 27 hindlimb muscles of the cat were digitized from transverse sections of spinal cord spanning the entire lumbosacral enlargement from the caudal part of $\mathrm{L}_{4}$ to the rostral part of $\mathrm{S}_{1}$ segments. Outlines of the spinal cord gray matter were also digitized. Models of the spinal cord were generated from these digitized data and displayed on a computer screen as threedimensional (3-D) images. We compiled a chart of electromyographic (EMG) profiles of the same 27 muscles during the cat step cycle from previous studies and used these to modulate the number of active MNs in the 3-D images. The step cycle was divided into 100 equal intervals corresponding to about $7 \mathrm{~ms}$ each for gait of moderate speed. For each of these 100 intervals, the level of EMG of each muscle was used to scale the number of dots displayed randomly within the volume of the corresponding $\mathrm{MN}$ pool in the digital model. One hundred images of the spinal cord were thereby generated, and these could be played in sequence as a continuous-loop movie representing rhythmical stepping. A rostrocaudal oscillation of activity in hindlimb MN pools emerged. This was confirmed by computing the locus of the center of activation of the MNs in the 100 consecutive frames of the movie. The caudal third of the lumbosacral enlargement showed intense MN activity during the stance phase of locomotion. During the swing phase, the focus of activation shifted abruptly to the rostral part of the enlargement. At the stance-swing transition, a transient focus of activity formed in the most caudal part of the lumbosacral enlargement. This was associated with activation of gracilis, posterior biceps, posterior semimembranosus, and semitendinosus muscles. These muscles move the foot back and up to clear the ground during locomotion, a role that could be described as retraction. The spatiotemporal distribution of neuronal activity in the spinal cord during normal locomotion with descending control and sensory inputs intact has not been visualized before. The model can be used in the future to characterize spatiotemporal activity of spinal MNs in the absence of descending and sensory inputs and to compare these to spatiotemporal patterns in spinal MNs in normal locomotion.

\section{IN T R O D U C T I O N}

It has been known for centuries that for a short time after cervical spinalization, many animals can still generate a loco-

Address for reprint requests: A. Prochazka, Center for Neuroscience, 507 HMRC, University of Alberta, Edmonton, Alberta T6G 2S2, Canada (E-mail: arthur.prochazka@ualberta.ca). motor rhythm (Mettrie de La 1745). T. Graham Brown coined the term "intrinsic factor" to describe the rhythm generator in the spinal cord and suggested a simple "half-center" oscillator as the underlying mechanism (Brown 1911). Brown's intrinsic factor was renamed the "central pattern generator" (CPG) (Grillner and Zangger 1975). Many experiments have been done in different species to localize and characterize the basic neuronal machinery of locomotor CPGs (Kiehn et al. 1998). Broadly speaking, opinion is split between those who believe that the vertebrate CPG is fairly localized (Cazalets et al. 1995) and those who think it is distributed (Arshavsky et al. 1997; Deliagina et al. 1983; Kiehn and Kjaerulff 1998; Kremer and Lev-Tov 1997). One version of the "distributed" hypothesis envisions a series of semi-autonomous "unit" oscillators distributed along the neuraxis and dynamically coupled by propriospinal pathways (Grillner and Wallen 1985). A consensus seems to be emerging that rhythmogenesis is more robust in one or two spinal cord segments just rostral to the lumbosacral enlargement than in more caudal segments and that these rostral segments may "lead" the other segments in generating the locomotor rhythm (Arshavsky et al. 1997; Deliagina et al. 1983; Kiehn and Kjaerulff 1998; Marcoux and Rossignol 2000).

It is impossible with current techniques to measure directly the activity of large ensembles of neurons of a particular functional type in the mammalian spinal cord during normal behavior. Although this may be possible in the future by identifying neurons according to some feature such as size, molecular affinity, or luminance, to our knowledge, this has not been attempted to date. Consequently, all of the experiments on localization of pattern generators within the spinal cord have been performed on reduced preparations of different types, including isolated embryonic spinal cords of the rat (Lev-Tov and O'Donovan 1995), in vitro portions of fetal rat spinal cords (Hochman et al. 1994; Kiehn and Kjaerulff 1998), and spinalized fictive locomotor cat (Andersson et al. 1978; Deliagina et al. 1983; Grillner and Zangger 1979) and lamprey (Grillner et al. 1998; Zhang et al. 1996). In these various experimental situations, the locomotor rhythm is usually initiated and maintained by the application to the spinal cord of pharmacological agents such as dihydroxy-phenylalanine (DOPA) or $N$-methyl-

\footnotetext{
The costs of publication of this article were defrayed in part by the payment of page charges. The article must therefore be hereby marked "advertisement" in accordance with 18 U.S.C. Section 1734 solely to indicate this fact.
} 
D-aspartate (NMDA) or by electrical stimulation applied to descending or peripheral sensory pathways. The rhythms generated in muscle nerves or ventral roots can often have many of the specific characteristics of those seen in normal locomotion, so the assumption is made that the patterns of central neural activity studied in this way are similar to those occurring in normal behavior. However, not only are descending inputs absent in these preparations, but in most cases, so are sensory inputs from actively moving limbs. Descending control originating in cerebral, cerebellar and midbrain areas has a profound influence on the amplitude and timing of muscle contractions during locomotion (Armstrong and Marple-Horvat 1996; Rho et al. 1997). Step-cycle phase transitions, as well as modulation of motoneuron (MN) activity during swing and stance phases, depend heavily on sensory input during normal locomotion (Grillner 1975; Grillner and Rossignol 1978; Pearson 1995; Prochazka 1996).

The aim of our study was to characterize the spatiotemporal migration of neuronal activity in the cat spinal cord during normal locomotion. We combined two sets of data: the known distribution of MN pools innervating different muscles within the spinal gray matter and the known EMG activity profiles of these muscles in the locomotor step cycle.

The approximate rostrocaudal distribution of $\mathrm{MN}$ pools in the cat spinal cord was originally deduced from the distribution of ventral roots innervating different hindlimb muscles (Sherrington 1892). Several important studies have greatly extended the scope and accuracy of this information (Romanes 1951; VanderHorst and Holstege 1997). In addition, there is now a large body of information on the location and reflex connections of interneurons of different types (Jankowska 1992) and on the distribution of the terminals of sensory axons (Brown 1981).

Each action potential in a MN propagates along the efferent axon and gives rise to a motor unit action potential (MUAP) in the muscle the MN innervates. All of the MUAPs generated by the numerous active MNs sum in a slightly nonlinear manner (see DISCUSSION) to produce the recordable signal called the electromyogram (EMG). The rectified EMG thus provides an indirect measure of the net firing of MNs of that muscle in the spinal cord at any particular moment. In numerous functional studies over the last three decades, the EMG patterns of cat hindlimb muscles recorded during locomotion have been described in great detail (see METHODS).

The model of MN distributions in the spinal cord and the model of spatiotemporal activity described in this paper are in digital form. This will allow them to be used in other laboratories not only to test for differences in spinal activity patterns in different preparations (e.g., fictive locomotor vs. normal), but also to help predict which muscles will be activated or inactivated by local electrical stimulation of the spinal cord (Mushahwar and Horch 1998; Mushahwar et al. 2000), lesions, or local excitation or inhibition with microinjections of pharmacological agents (Marcoux and Rossignol 2000). A preliminary report on this work has been published (Yakovenko et al. 2000).

\section{METHOD S}

\section{Digitization}

In a recent study, the horseradish peroxidase (HRP) staining technique was used to determine the distributions within the cat spinal cord of $50 \mathrm{MN}$ pools innervating hindlimb, pelvic floor, and lower back muscles (VanderHorst and Holstege 1997). The entire lumbosacral enlargement of the frozen spinal cord (mean length: $28.8 \mathrm{~mm}$ ) was cut into $40-\mu \mathrm{m}$ sections. Every second section was collected. Groups of 12 such sections were used to construct composite digital drawings showing the positions of all stained MNs in the group (i.e., each drawing corresponded to a $12 \times 2 \times 0.04=0.96-\mathrm{mm}$ thick slice of spinal cord). The rostral and caudal ends of the enlargement were identified on the basis of distinctive transitions in shape of the ventral horn. Because the enlargements varied in length between animals, a normalization procedure was used to divide each enlargement into 100 levels. A summary figure of 30 slices was presented (VanderHorst and Holstege 1997, Fig. 28), corresponding to a mean length of the enlargement of $30 \times 0.96=28.8 \mathrm{~mm}$.

Based on the EMG profiles available to us (see following text), we digitized the positions of MNs of the following 27 hindlimb muscles: adductor femoris magnus (AdFM), biceps femoris anterior and posterior $(\mathrm{BFa}, \mathrm{BFp})$, caudofemoralis $(\mathrm{CF})$, extensor digitorum longus (EDL), flexor digitorum longus (FDL), flexor hallucis longus (FHL), gluteus maximus and medius (GlutMax, GlutMed), gastrocnemius lateralis and medialis (LG, MG), gracilis, iliacus, plantaris, psoas, rectus femoris (RF), sartorius anterior and sartorius medialis (SRTa, SRTm), soleus, semimembranosus anterior and posterior (SMa, SMp), semitendinosus (ST), tibialis anterior (TA), tibialis posterior (TP), vastus intermedius, vastus lateralis, and vastus medialis (VI, VL, VM).

Initially we classified MNs as innervating either flexors or extensors, according to the predominant biomechanical action of the corresponding muscles or parts of muscles activated in the flexor and crossed extensor reflexes (Carrasco and English 1999; Eccles and Lundberg 1958; Sherrington 1910). Flexors: BFp, EDL, gracilis, iliacus, psoas, RF, SMp, SRTa, SRTm, ST, TA; extensors: AdFM, BFa, FDL, FHL, GlutMax, GlutMed, LG, MG, plantaris, soleus, SMa, TP, VI, VL, VM. However, we will argue later that in locomotion $\mathrm{BFp}, \mathrm{SMp}$, and ST muscles, and possibly gracilis and FDL form a separate functional group and their MNs produce a distinct locus of activation in the spinal cord.

In the anatomical study (VanderHorst and Holstege 1997), two characteristics of the spinal cord were recognized when determining the spatial relation between lumbosacral MN pools. First, there were major differences in the organization of spinal rootlets between cats and so segmental localization varied. Second, the length of the lumbar enlargement varied considerably [mean: $28.8 \pm 2.4(\mathrm{SD}) \mathrm{mm}$, range: 23.0-34.1 $\mathrm{mm}(n=120)]$. To normalize the data across animals, two easily recognizable landmarks were chosen to identify the rostral and caudal ends of the enlargement. The rostral landmark (defined as level 0 ) was a sudden lateral extension or bulging of the ventrolateral corner of the ventral horn into the white matter in the midlumbar cord. The caudal landmark (defined as level 100) was in the sacral cord where the border of the ventral horn changes from a rounded profile to a straight line joining the ventromedial and dorsolateral corners of the horn. These changes in shape at levels 0 and 100 took place over a distance of 0.5-1.0 mm (approximately 1.5-3 levels). Locating them accurately required careful comparisons of several sections in the vicinity of the transitions. The 0 - to 100-level scale allowed the relative distributions of many $\mathrm{MN}$ pools to be compared. In the numerous case studies illustrated (VanderHorst and Holstege 1997), the rostrocaudal locations of the centers of mass of individual MN pools ranged over maximally 10 levels (i.e., localization was reliable to within $10 \%$ of the length of the enlargement). It should be noted that this normalization procedure was seen as an important advance over previous methods, in which relative positions of MN pools could only be studied accurately in individual animals.

To enter the positions of MNs into the model, we scanned Fig. 28 of VanderHorst and Holstege (1997), at high resolution $(600 \times 600$ dpi). A software program written in Matlab (version 5.3, The MathWorks) was used to display each scanned slice on a 17-in computer monitor and to digitize the coordinates of individual MNs together 
with the outline of the gray matter. The coordinates, stored in ASCII format, were imported into a three-dimensional (3-D) model constructed with a second Matlab software program. In the model, the slices were all represented as being $0.96-\mathrm{mm}$ thick and accordingly the length of the enlargement was represented as $28.8 \mathrm{~mm}$. It should be stressed that in the original study lumbar enlargements varied in length over the range 23.0-34.1 mm (VanderHorst and Holstege 1997). In this paper, we will therefore usually refer to the 100 normalized levels; however, certain calculations such as that of the center of activation require absolute positions. The slices were aligned using the central canal for lateral centering and the midline between dorsal and ventral horns for dorsoventral centering. As the dimensions of individual cross-sections varied, the absolute positions of MNs and the boundaries of MN pools in the slices can only be considered accurate to within the variance of the original dimensions. In unpub- lished data from five cats, one of the authors (V. K. Mushahwar) made measurements of the width and thickness of the spinal cord at different segmental levels. The mean distance from the midline to edge of the white matter at $\mathrm{L}_{7}$ level was $3.6 \pm 0.04 \mathrm{~mm}$ and the corresponding mean dorsoventral thickness of the spinal cord was $5.5 \pm 0.1 \mathrm{~mm}$. As most MNs were located about halfway from the midline to the edge of the white matter and less than half of the thickness of the cord from the central canal, we estimate SDs of the absolute positions of MNs to be no more than $0.02 \mathrm{~mm}$ anterolaterally and $0.05 \mathrm{~mm}$ dorsoventrally.

The Matlab functions for visualization of 3-D structures were used to generate a 3-D model of the lumbar enlargement. Figure 1 shows graphical displays of this model in which surfaces have been "rendered" with SURFdriver version 3 software (Surfdriver.com).

EMG patterns of cat limb muscles have been studied extensively by many researchers (Abraham et al. 1985; Belanger et al. 1988; Buford

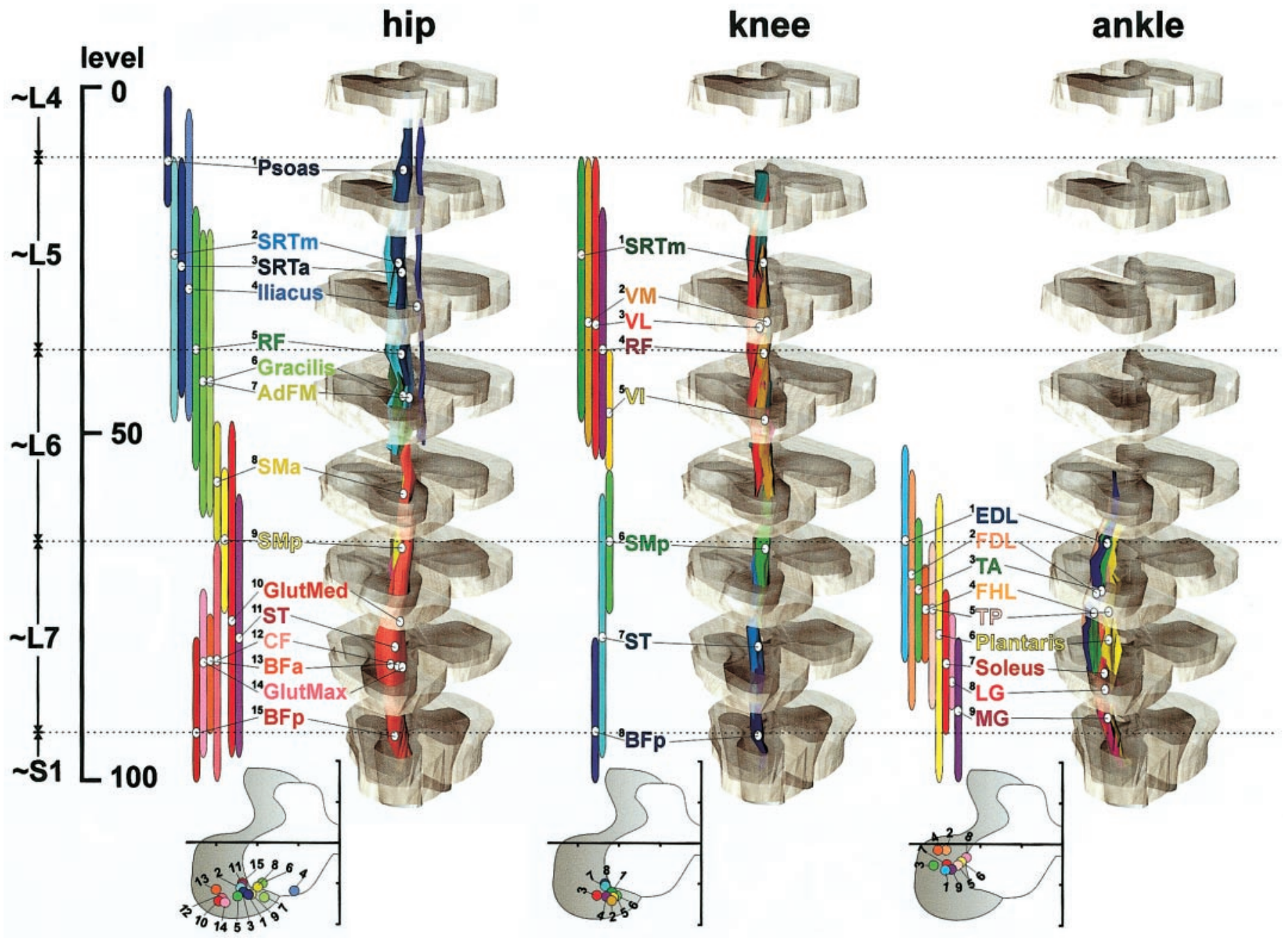

FIG. 1. Anatomical models of the organization of the motoneuron $(\mathrm{MN})$ pools within the cat lumbar enlargement. The pools innervating muscles acting about the hip (left), knee (middle), and ankle (right) are presented separately. The anatomical data were digitized from 30 summary drawings or "slices" (VanderHorst and Holstege 1997). The rostrocaudal axis is normalized and divided into 100 levels. On average slices were $0.96-\mathrm{mm}$ thick and corresponded to 3.3 levels. The models show a structure $29 \mathrm{~mm}$ long on average, spanning the caudal $\mathrm{L}_{4}$ to rostral $\mathrm{S}_{1}$ segments of the spinal cord. The fusiform $\mathrm{MN}$ pools within the gray matter are long and intertwined (MN pools are identified by color, abbreviation, and a number in each of the 3 representations). Left: the schematic bars show their rostrocaudal extent. $\bigcirc$, the centers of mass; bottom: their positions in the transverse plane. The lines leading from the abbreviations point to the location of the corresponding center of mass. Note the rostral locations of hip flexor pools [Psoas, sartorius (SRT), iliacus] and more caudal locations of hip extensor pools [e.g., semimembranosus anterior (SMa) and biceps femoris anterior $(\mathrm{BFa})]$. The reverse relationship is true of $\mathrm{MN}$ pools innervating knee muscles: knee flexor MN pools [e.g., biceps femoris posterior (BFp) and semitendinosus (ST)] are more caudal and knee extensor MN pools [e.g., vastus lateralis (VL) and rectus femoris $(\mathrm{RF})$ ] more rostral. Ankle MN pools are relatively close together in the caudal region, with flexors slightly more rostral than extensors. BFp, semimembranosus posterior (SMp), and ST are bifunctional muscles that flex the knee and extend the hip, so their MN pools are represented in both middle and left. 
and Smith 1990; Carrier et al. 1997; Engberg and Lundberg 1969; Halbertsma 1983; Pratt et al. 1991; Wand et al. 1980). In a previous paper from this laboratory, we reviewed the available data and constructed a chart of standardized EMG profiles of a variety of muscles in the cat step cycle (Prochazka et al. 1989). In the present study, we again reviewed all the currently available literature and collated as many examples as possible of segments of EMG locomotor recordings from hindlimb muscles. From this collection, we constructed EMG profiles representing the activity in one step cycle for each of 27 muscles. We considered doing this mathematically by digitizing and processing the individual traces from the different studies to produce averages aligned to foot contact and normalized to one cycle period; however, we decided against this for the following reasons. The EMG recordings were not of uniform quality or format. Some EMG signals were rectified and smoothed while others were unrectified. Some were averages of several steps while others were raw traces from single cycles. Some recordings included reliable measurements of ground contact and foot lift while others did not. Cycle durations varied and as a result so did the relative durations of swing and stance phases. Even when EMG recordings are made in the same laboratory with the same techniques there is some variation in the time course of EMG profiles from a given muscle that appears to depend on electrode dimensions and placement as well as possible differences in gait between animals. Taking all of these factors into consideration, we therefore opted to construct each profile from linear segments after carefully comparing the available records in the collection and using our judgement on critical features such as the timing of onset and duration of the EMG signal, the timing of the peaks, and the overall shape of the signal. Profiles were constructed from a number of piecewise linear segments, the onset, duration, and slope of which were entered digitally in a standardized spreadsheet of the step cycle in which the landmarks were the moments of foot lift and foot contact. The swing phase occurring between these landmarks was standardized to $30 \%$ of the cycle, a proportion associated with gait velocities in the range $0.6-0.8 \mathrm{~m} / \mathrm{s}$ and cycle periods of about $700 \mathrm{~ms}$ (Goslow et al. 1973; Halbertsma 1983). Particular attention was paid to the time course of EMG signals at and around these critical points in the cycle. When possible, concurrent EMG signals from ankle extensors MG, LG, or soleus were used as a reference for foot contact, as the mean delay between the onset of EMG in these muscles in the E1 phase and the moment of foot contact is $60 \mathrm{~ms}$, with a range of $\pm 5 \mathrm{~ms}$ (Prochazka and Gorassini 1998; Trend 1987). Muscle length or joint angle traces, when available, also helped us to identify timing landmarks. Each profile was normalized, i.e., it reached a peak of $100 \%$ at least once in the cycle. The assumption implicit in this normalization is that all muscles are fully recruited at least once in the step cycle. Clearly this does not occur in slow locomotion (Walmsley et al. 1978). However, we viewed this simply as a matter of scaling; the important point was that all MN pools, whether flexor, extensor, or retractor, reached the same maximal level of recruitment when their EMG signals were maximal. Different relative levels of recruitment of flexors and extensors were then explored to address the issue of parametric sensitivity.

The step cycle was divided into 100 equal intervals corresponding to about $7 \mathrm{~ms}$ each for gait of moderate speed. For each of these intervals, the normalized level of EMG of each hindlimb muscle was obtained from the corresponding profile in the chart. The EMG levels from each muscle were used to scale the number of dots displayed within the volume of the corresponding MN pool in the digital model. This assumes a linear relationship between EMG and MN firing rate (Hoffer et al. 1987b), an assumption we discuss in detail later. To simplify the simulation, we also assumed a random topographical distribution of active MNs within each MN pool. The 100 consecutive images or frames were saved in bitmap format, and when displayed in rapid sequence with Corel Photo-Paint software, they formed a continuous-loop movie showing the spatiotemporal activity of hindlimb MN pools during locomotion.
The effect of our simplifying assumptions on the results and conclusions will be dealt with in more detail in the DISCUSSION . However, to gain an overall idea of the parametric sensitivity of our main conclusions to details of EMG profiles, we did a set of simulations in which the profiles were converted to all-or-nothing step functions such that all nonzero values were assigned the value $100 \%$.

\section{Calculation of the center of MN activity}

The locus of MN activity was analyzed by calculating the center of mass $\left(r_{\mathrm{cm}}\right)$ of the $n$ active MNs (the "center of activity") at each of the 100 moments in the step cycle, using the following formula

$$
r_{\mathrm{cm}}=\frac{\sum_{i=1}^{n} r_{i}}{n}
$$

where $r_{i}$ is the vector from the origin to the $i$ th $\mathrm{MN}$ (the origin being defined as the rostral-most point of the neuraxis). As mentioned in the preceding text, the center of activity was expressed in terms of absolute position within the standard 28.8-mm long enlargement.

\section{R E S U L T S}

\section{Anatomical distribution of MN pools in the lumbosacral spinal cord}

Figure 1 shows three views of the 3-D model of the lumbar enlargement of the cat spinal cord rendered from the digitized transverse slices of HRP-stained MNs described in the preceding text. Each of the three displays shows the outlines of the gray and white matter as well as $\mathrm{MN}$ pools of muscles acting about the hip, knee, and ankle respectively. The figure illustrates some interesting general features of $\mathrm{MN}$ pool distributions. The pools are long, spindle-shaped, and intertwined. The bars on the left of each model were included to show the rostrocaudal extent and the center of mass of each pool. As is well known from classical myotomal charts (Romanes 1951; Sharrard 1955), there is a correspondence between the rostrocaudal position of $\mathrm{MN}$ pools and the anatomical position of their respective muscles in the leg. It follows that there must be a correlation between the position of MN pools and the biomechanical action with which they are associated. Thus starting at level 0 at the rostral end of the enlargement and moving caudally to level 80 , we find the MN pools of muscles distributed in proximal to distal order in the anterior compartment of the leg: hip flexors (psoas, SRTm, SRTa, iliacus) knee extensors (VL, VM, RF, VI), and ankle flexors (EDL, TA). When the leg is pendant, these muscles all act to move the foot forward. Starting from level 40 and moving caudally to level 100 ( $S_{1}$ segment), we find the hip extensor pools (AdFM, gracilis, SMa, SMp), followed by toe plantarflexors (FDL, FHL) and ankle extensors (MG, LG, Pl, soleus). The most caudal part of the enlargement also contains $\mathrm{MN}$ pools of proximal muscles with complex actions about hip and knee (GlutMed, ST, CF, BFa, BFp). When the leg is pendant, these muscles all assist in moving the foot and toe back. Note that in terrestrial locomotion, some of the muscles assume a loadcompensating role that can no longer be described in terms of a simple forward-backward dichotomy (see DISCUSSION).

The numerical distributions of the digitized MNs are illustrated in Fig. 2. There is a significant imbalance between the sizes of rostral and caudal populations of MNs. The caudal half of the enlargement (level 50-100) contains far more MNs than 


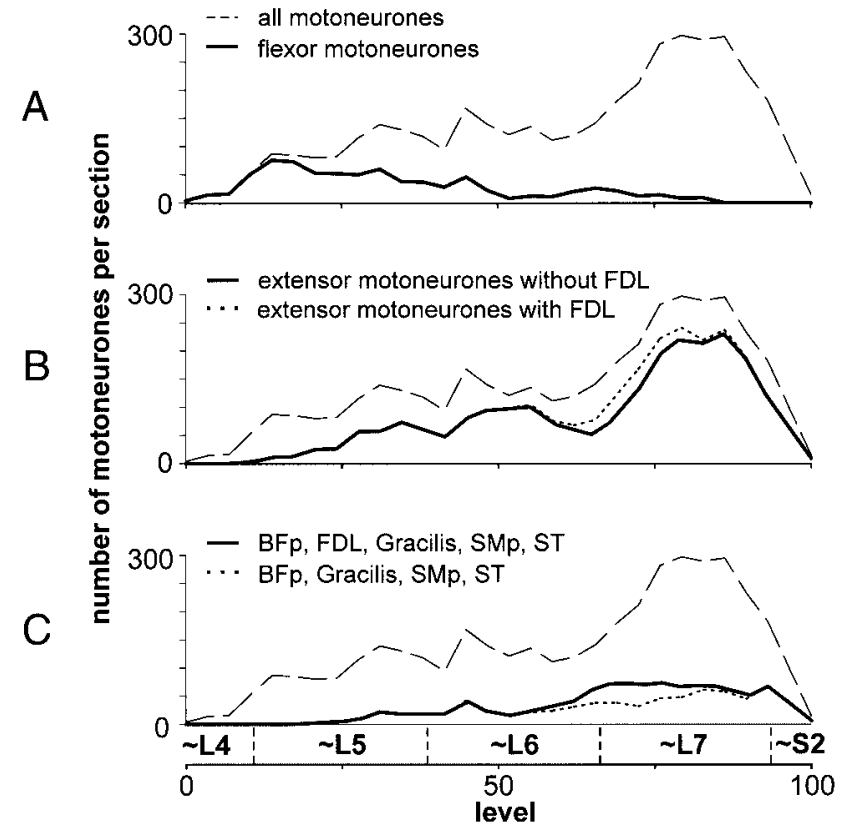

FIG. 2. MN distribution along the rostrocaudal axis of the lumbar enlargement of the cat spinal cord. The enlargement was divided into 30 slices as described in the text. The number of MNs digitized from the source data in each slice is plotted. As no distinction was made between alpha and gamma MNs, the number of alpha MNs is probably overestimated (see text). The total population distribution is shown as a dashed line in each panel and reveals a significant imbalance between the rostral and caudal halves of the lumbosacral enlargement. $A$ : flexor MNs are concentrated mainly in the rostral part of the spinal cord (levels $0-50$ ). $B$ : extensor MNs are more caudal, with peak density in the $\mathrm{L}_{7}$ segment. Two distributions are shown, 1 that includes flexor digitorum longus (FDL) MNs ( $\cdots$ ) and 1 that does not (-: see text). $C$ : distribution of MNs innervating BFp, gracilis, SMp, and ST (with and without FDL). We argue in the text that these muscles, normally classified as flexors, have a biomechanical role in the step cycle that is best described as retraction.

the rostral half. Indeed it is well known that the caudal ventral horns have lateral extensions that accommodate the increased numbers of MNs (VanderHorst and Holstege 1997). Most of the MNs in this region innervate extensor muscles (Fig. 2B). Over $75 \%$ of all hindlimb extensor MNs are in this region (Table 1) and their density reaches a peak at about level 80. Flexor MNs (Fig. 2A) are less tightly clumped, but even so, more than $80 \%$ of them are found in the rostral half. The distributions of BFp, FDL, gracilis, SMp, and ST MNs are illustrated separately in Fig. 2C. These MNs are mainly in the caudal half of the enlargement. We will argue that the function of these muscles in locomotion is to provide the final extensor thrust during the stance phase and then to provide ground clearance for the swing phase by extending the hip while at the same time flexing the knee and the toes. For this reason, we have borrowed the term "retractors" from the invertebrate literature to describe them (see DISCUSSION).

\section{Analysis of the spatiotemporal pattern of MN activity}

Figure 3 summarizes the EMG profiles of all the muscles we included in the model during level forward walking, starting from the onset of swing (see METHODs for details on how the profiles were constructed). The muscles in each group behave rather uniformly during locomotion. The flexors are active mainly in the early and mid-swing phase when the foot is off the ground. The extensors become active during the last 100 $\mathrm{ms}$ of swing. In fact, this part of the step cycle is usually designated E1, the first extensor phase of the step cycle (Philippson 1905). The bifunctional muscles BFp, gracilis, and ST show two EMG bursts in the step cycle, at the transitions from stance to swing and from swing to stance respectively (SMp does not always show this burst clearly). These muscles produce a backward and upward motion of the foot at the end of the stance phase, a function we will argue in the following text is best described as retraction. FDL may contribute to this action by plantarflexing the paw. FDL was originally considered an extensor, along with its synergist FHL (Sherrington 1910). Several EMG studies supported this view, showing FHL and FDL to have extensor bursts similar to those of the gastronemii and soleus (Abraham et al. 1985; Belanger et al. 1996; Duysens and Loeb 1980; Engberg 1964; Forssberg 1979; Rasmussen et al. 1978). However, in a study that focused specifically on the biomechanical actions and EMG of FDL and FHL, it was concluded that unlike FHL, FDL acted predominantly as a toe flexor at the stance-swing transition with a brief, intense burst of EMG (O'Donovan et al. 1982). It was suggested that the EMG during the stance phase recorded by others had been due to cross-talk. The flexor burst pattern of FDL is supported in neurograms recorded during fictive locomotion (Fleshman et al. 1984) and in more recent EMG recordings in normal cats (Loeb 1993; Smith et al. 1998; Trank and Smith 1996), although Loeb's group reported variable amounts of stance phase activity that was unlikely to be due to cross-talk. Given the ambiguity regarding FDL in the literature, we performed two separate analyses of the data, in one case assuming a flexor (retractor) burst EMG profile for FDL and in the other an extensor profile (see dashed profiles in Fig. 3).

We were unable to include some muscles with MNs in the lumbar enlargement for the following reasons. There were no recordings from intrinsic hip muscles such as obturator, pyriformis, and gemellus in the literature. Tensor fascia latae, a thin, flat muscle overlying the vasti, has anterior and posterior portions that flex or extend the hip respectively (Chanaud et al. 1991). These portions were not separated in the anatomical study (VanderHorst and Holstege 1997), and so EMG profiles could not be duly assigned. For the same reason, the small intrinsic muscles of the foot are omitted from our analysis. A case in point is extensor digitorum brevis. This muscle is

TABLE 1. Rostral $\left(L_{5}-L_{6}\right)$ and caudal $\left(L_{7}-S_{1}\right)$ populations of extensor, BFp-FDL-gracilis-SMp-ST and flexor MNs in the cat lumbar enlargement

\begin{tabular}{|c|c|c|c|c|}
\hline Rostral half $\left(\mathrm{L}_{4}-\mathrm{L}_{6}\right)$ & $549(23)$ & $155(17)$ & $620(81)$ & $1324(33)$ \\
\hline Full $\left(\mathrm{L}_{4}-\mathrm{S}_{1}\right)$ & 2366 & 907 & 761 & 4034 \\
\hline
\end{tabular}

Parentheses enclose percentages. BFp, biceps femoris posterior; FDL, flexor digitorum longus; SMp, semimembranosus posterior; ST, semitendinosus. 


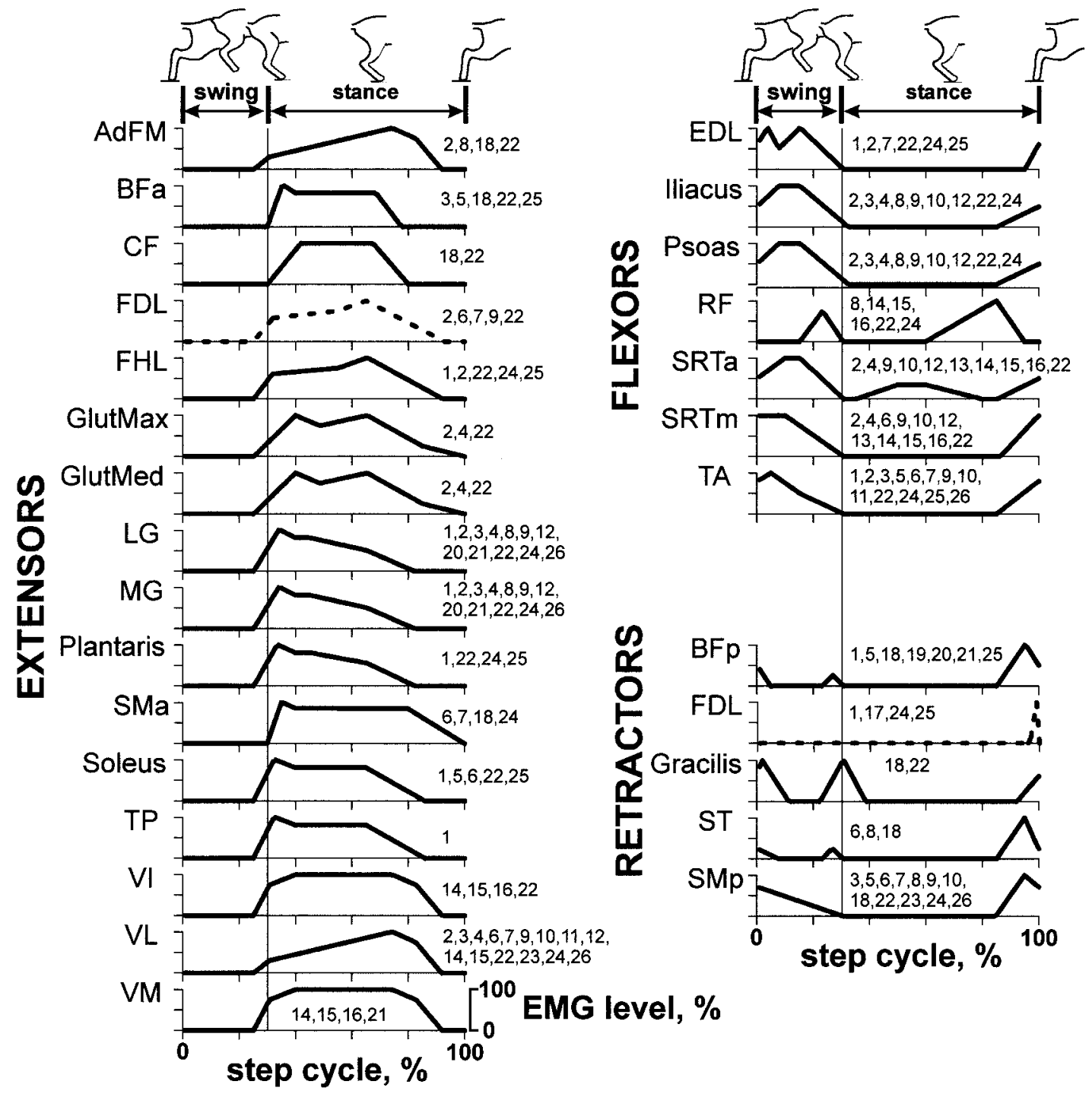

FIG. 3. EMG patterns of hindlimb muscles during forward level locomotion at moderate speeds (approximately $0.6 \mathrm{~m} / \mathrm{s}$ ). The normalized patterns are plotted for a full step cycle, starting from the onset of swing. Left and right: the EMG profiles of extensors and flexors and the muscles we classify as retractors, respectively. - - , FDL, which has a variable EMG pattern, shown in both extensor and retractor groups. These EMG profiles were constructed from piece-wise linear segments from numerous sources in the literature, the numbers in each panel referring to the papers listed here. The profiles were used in the subsequent figures to modulate the number of MNs shown as being active in the spatiotemporal model. References: 1) Abraham et al. (1985), 2) Belanger et al. (1996), 3) Buford and Smith (1990), 4) Carrier et al. (1997), 5) Chanaud et al. (1991), 6) Duysens and Loeb (1980), 7) Engberg (1964), 8) Engberg and Lundberg (1969), 9) Forssberg (1979), 10) Forssberg et al. (1980), 11) Gritsenko et al. (2000), 12) Halbertsma (1983), 13) Hoffer et al. (1987a), 14) Hoffer et al. (1987b), 15) Loeb and Hoffer (1985), 16) Loeb et al. (1985), 17) O’Donovan et al. (1982), 18) Pratt et al. (1996), 19) Pratt et al. (1991), 20) Prochazka et al. (1976), 21) Prochazka et al. (1989), 22) Rasmussen et al. (1978), 23) Rossignol et al. (1986), 24) Smith et al. (1998), 25) Trank and Smith (1996), 26) Wand et al. (1980).

innervated by branches of the deep peroneal nerve, and so its MNs are probably close to those of EDL, in caudal $\mathrm{L}_{6}$ and rostral $\mathrm{L}_{7}$ segments. EDB EMG shows a burst of activity at the onset of the stance phase (Trank and Smith 1996), so this might slightly alter the activity in our model. Given the likely small number of MNs involved, this is unlikely to be a major effect.

Conversely, although the distribution of MNs of the minor portions of adductor femoris (brevis and longus) were available, the only EMG recordings in the literature were from the major portion (magnus), so the minor portions are not represented in our model.

The MN distributions of the axial muscles multifidi and longissimus dorsi showed a sparse population between $\mathrm{L}_{6}$ and $\mathrm{S}_{1}$ and included some tail MNs at the caudal end (VanderHorst and Holstege 1997). The only EMG recordings from these two muscle groups we could find in the literature were made at $\mathrm{L}_{4}$ level in the normal cat (Carlson et al. 1979) or at $\mathrm{L}_{4}$ and $\mathrm{L}_{7}$ levels in the decerebrate or spinal cat (Zomlefer et al. 1984). In the normal cats, for locomotion at about $1 \mathrm{~m} / \mathrm{s}$, these muscles showed biphasic, low-level EMG in both swing and stance phases of the step cycle. In the decerebrate cats, for locomotion slower than $0.7-0.8 \mathrm{~m} / \mathrm{s}$, there was virtually no detectable EMG signal in the vertebral muscles (Zomlefer et al. 1984). To gain some perspective on this issue, we obtained EMG record- 
ings from the longissimus dorsi muscle between the $\mathrm{L}_{5}$ and $\mathrm{L}_{6}$ spinous processes in one cat with the use of percutaneous electrodes. The EMG pattern was low level and fairly constant over the step cycle, which agrees with the preceding observations. In light of the incomplete nature of the data on vertebral muscles, it did not seem appropriate to include these in the analysis. Given their small numbers and aparently low level of recruitment in slow stepping, their omission is unlikely to affect our main conclusions, although it would of course be desirable to confirm this if and when more EMG and anatomical data become available.

The main focus of this study, the spatiotemporal pattern of the MN activity during locomotion, is illustrated in Fig. 4. The plot shows the distribution of the active MNs along the rostrocaudal axis in two full step cycles. As described in METHODS, the step cycle was divided into 100 equal intervals and for each interval, the normalized EMG level of each muscle in Fig. 3 was used to compute the number of active MNs per slice [a slice corresponds to approximately $1 \mathrm{~mm}$ of spinal cord or 3.3 normalized levels (VanderHorst and Holstege 1997)]. Figure 4 reveals a large imbalance in MN activity in swing and stance phases, the latter associated with the large number of extensor $\mathrm{MNs}$ in the caudal half of the enlargement. Note the relatively small size of the rostral peak of activity centered between $\mathrm{L}_{4}$ and $\mathrm{L}_{5}$ in the swing phase.

The movie animation of Fig. 4 (which may be obtained in digital form from the authors) created the impression of a rostrocaudal oscillation during the step cycle. This was confirmed by computing and plotting the rostrocaudal movement of the center of activity in a plan view of the same data (Fig. $5 A$ ). The locus of the center of MN activity (open circles) was calculated in equal $1 \%$ increments of the cycle period, (corresponding to 7-ms intervals for a 700-ms cycle period typical of medium-speed locomotion). The distance between the open circles is proportional to the velocity of the center of activity; the velocity is maximal at the transition from swing to stance. During the stance phase, there is a period in which the center of activity dwells in the region about level 70 . Note that the vertical scales in the panels of Fig. 4 and 5 vary because we normalized to the peak number of active MNs per slice in each case so as to use the full range of the color spectrum.

The large stance phase peak centered between $\mathrm{L}_{6}$ and $\mathrm{L}_{7}$ in Fig. 4 is followed by a smaller peak of activation in the $L_{7}$ segment associated with activity of FDL, BFp, gracilis, SMp, and ST MN pools at the transition from stance to swing. This peak also shows up clearly in the plan view in Fig. $5 A$. For a brief instant in this phase of the step cycle, this is virtually the only part of the whole lumbosacral enlargement with active MNs. The transitions from swing to stance and retraction to swing are abrupt events in which the focus of activity jumps from one end of the enlargement to the other: the computed center of activity moves between levels 36 and 74, i.e., 38 levels, equivalent to $10.9 \mathrm{~mm}$ in the average-sized enlargement. As the centers of activation of the most proximal and distal MN pools (Psoas and BFp) are at 4.3 and $25.8 \mathrm{~mm}$, respectively, in the average enlargement, the maximal possible displacement of the center of activity is on average $21.5 \mathrm{~mm}$, i.e., 75 levels. Thus the center of activity moves $51 \%$ of its maximal possible range. The swing-stance transition is a little faster than the transition from retraction to stance: the center of activity reaches a peak velocity of $361 \mathrm{~mm} / \mathrm{s}$ in the swingstance transition and $309 \mathrm{~mm} / \mathrm{s}$ during the retraction-swing transition. The figure also shows up a transient spread of activity rostrally to slice $11-15$ about two-thirds of the way through the stance phase. This transient is associated with EMG activity of VL and RF muscles. Like VL, RF extends the knee but it also flexes the hip. Its activation is followed immediately by that of its antagonists, BFp, gracilis, SMp, and ST, with an associated caudal shift of the center of activation in the spinal cord.

The EMG profiles on which Figs. 4 and $5 A$ were based were derived from many studies, and their construction involved qualitative judgements and approximations. How sensitive are such data to parametric variations in the EMG profiles? Three

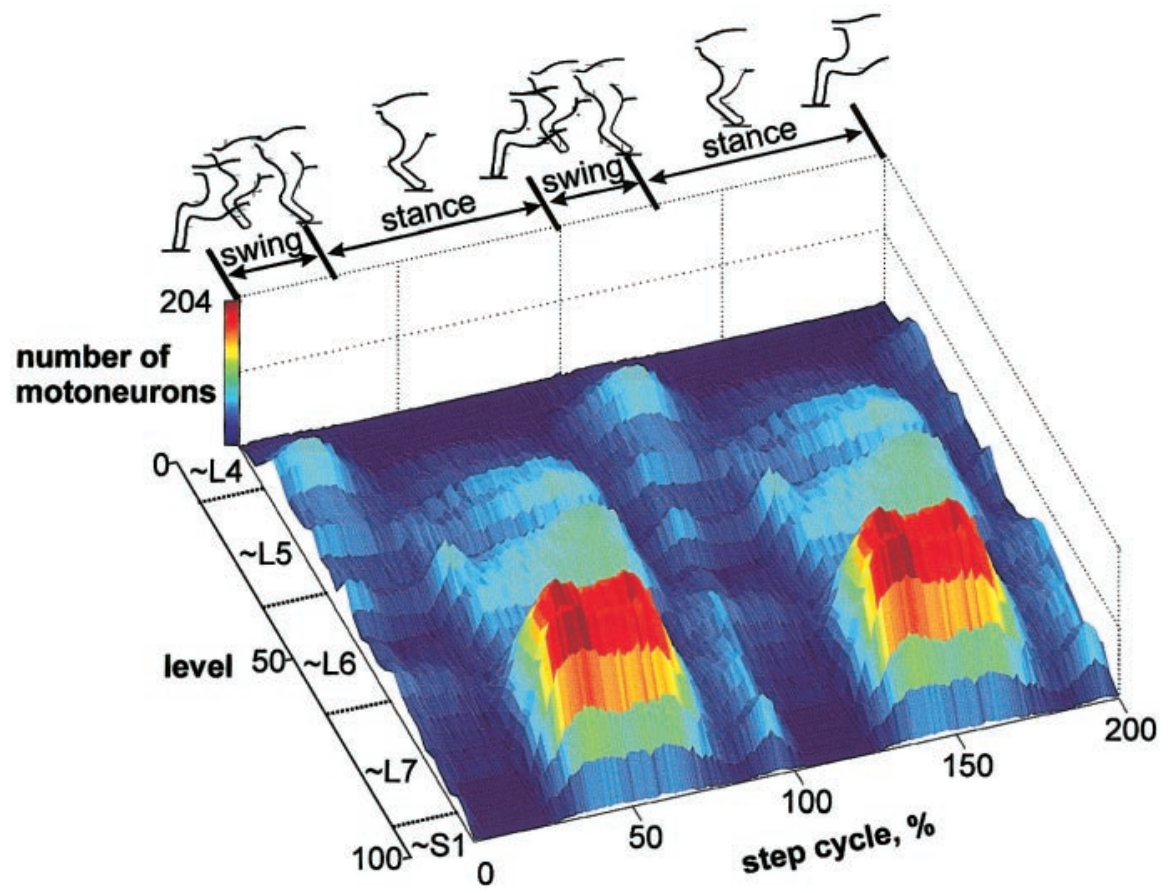

FIG. 4. Modeled spinal MN activity in the lumbosacral enlargement during locomotion. The digitized MN distributions shown in Fig. 1 were combined with the EMG profiles shown in Fig. 3 to produce modeled activation patterns of MNs during 2 step cycles of forward, level locomotion [in this figure and the next, FDL was assigned the flexor (retractor) profile of Fig. $3]$. The phases of the step cycle are indicated above the 3-dimensional plot. Each step cycle was divided into 100 time intervals corresponding to about $7 \mathrm{~ms}$ for slow walking. The plot shows the time course of activity of MNs in 30 transverse slices of the cord [level 0, most rostral; level 100, most caudal; each slice corresponds to approximately 3 levels or approximately $1 \mathrm{~mm}$ (VanderHorst and Holstege 1997)]. Note the dominant peaks of activity in the caudal slices in the stance phases and the relatively small rostral peaks during the swing phases. Note too the rapid migration of activity in the transition from swing to stance and a period between stance and swing where activity dwells in the caudal half of the enlargement. 
A flexors-retractors-extensors: $100 \%$ (graded)

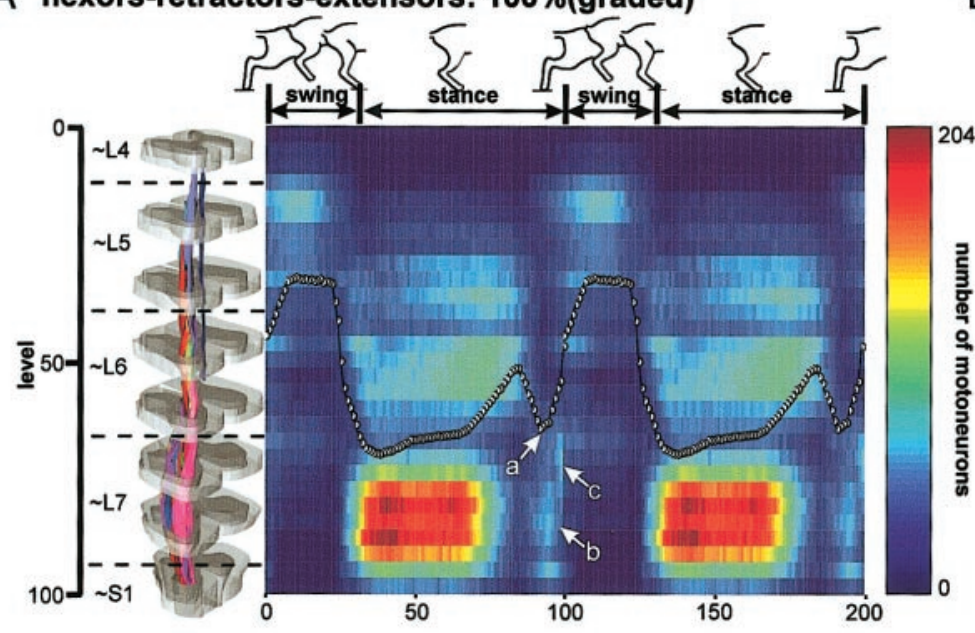

$B$ flexors-retractors-extensors: $100 \%$ (all-or-nothing)

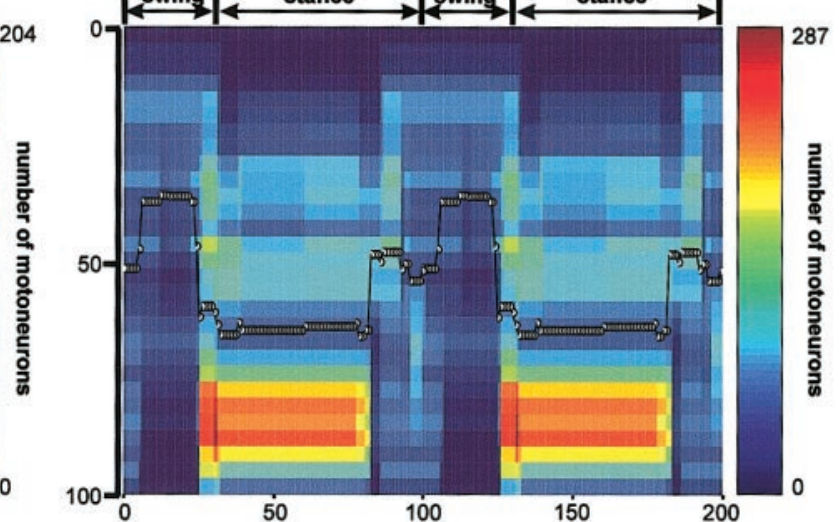

C flexors-retractors: $15 \%$, extensors: $\mathbf{4 0 \%}$ (graded)

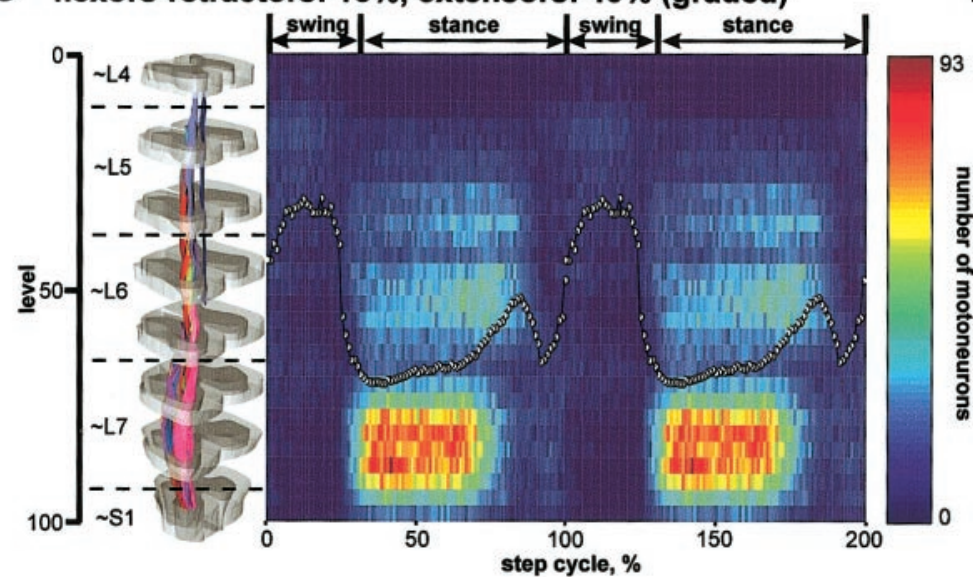

D flexors-retractors: $40 \%$, extensors: $15 \%$ (graded)

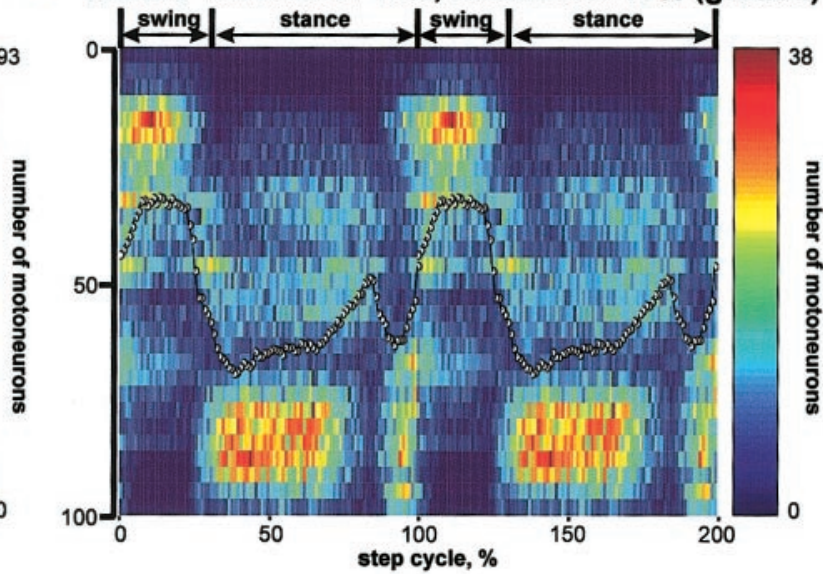

FIG. 5. Variations in assumed activation patterns to assess parametric sensitivity. A: graded recruitment reaching a maximum of $100 \%$ in all MN pools. This plot is a plan view of the plot in Fig. 4. O, the positions of the center of mass of net MN activity were calculated at each time increment of the step cycle. The migration of these centers of activity was most rapid at the swing-stance transition. At the stance-swing transition the center of activation moves caudally (arrow a), then rostrally again. This caudal detour is associated with activity in BFp, gracilis, SMp and ST (arrow b) and FDL (arrow c). B: all-or-nothing recruitment: the EMG profiles of Fig. 3 were converted to step functions such that all non- 0 values were assigned the value $100 \%$. These step functions were then used to modulate the numbers of active MNs in a given pool between 0 and $100 \%$. This resulted in activity plots that had similar general features to those in $A$, but transitions were more abrupt and the spatiotemporal migration of activity lost some of its subtleties. The caudal detour at the stance-swing transition was present although attenuated. $C$ : graded recruitment reaching a maximum of $15 \%$ in flexor and retractor $\mathrm{MN}$ pools and $40 \%$ in extensor MN pools. This is probably the case that most closely mimics real-life locomotion. (retractor profile used for FDL). $D$ : graded recruitment reaching a maximum of $40 \%$ in flexor and retractor $\mathrm{MN}$ pools and $15 \%$ in extensor $\mathrm{MN}$ pools. The general features of the rostrocaudal movement of the center of activity shown in $A$ were preserved in $B$ and $C$. The brief caudal detours of activity at the stance-swing transition are attenuated in $C$ and exaggerated in $D$. Note that because the total number of active MNs varied according to the assumptions, the color scales were adjusted to preserve contrast and detail in each plot.

parametric variations are illustrated in Fig. 5, $B-D$. In Fig. 5B, we eliminated incremental modulations in the amplitudes of EMG profiles and used only the timing information (i.e., the profiles of Fig. 3 were converted to all-or-nothing step functions such that all nonzero values were assigned the value $100 \%$ ). As may be seen, the main effect of this was to reduce the net displacements of activity and to create more abrupt changes at phase transitions. The caudal detour of activity at the stance-swing transition was reduced. However, the main conclusions drawn from Figs. 4 and $5 A$ still held.

In Fig. 5, $C$ and $D$, we assessed the sensitivity of the plots in Figs. 4 and $5 A$ to variations in the relative level of $\mathrm{MN}$ recruitment in flexor, extensor, and retractor muscles. Figure
$5 C$ shows the data of Fig. 4 recalculated on the assumption that extensor MNs were recruited to a maximum of $40 \%$ at peak EMG and flexor and retractor MNs were recruited to a maximum of $15 \%$ [ 40 and $15 \%$ were chosen on the basis of data comparing locomotion with more forceful movements such as paw-shakes in which recruitment approaches 100\% (Chanaud et al. 1991)]. These values were switched in Fig. 5D: flexor and retractor MNs were assumed to reach $40 \%$ recruitment at peak EMG and extensor MNs were assumed to reach a maximum of $15 \%$ recruitment. In all cases, the general features of the rostrocaudal movement of the center of activity shown in Fig. $5 A$ are preserved.

In a separate analysis, we replotted Fig. $5 A$ on the assump- 
tion that FDL had the extensor activity profile shown in Fig. 3 $(l e f t)$. This was performed in case the inclusion of FDL as a retractor may have given undue weight to the caudal detour of activity at the stance-swing transition. The plot obtained was hard to distinguish from that of Fig. 5A. We conclude that the stance-swing transition does indeed represent a distinct phase of migration of MN activity associated with retraction.

\section{I S C U S S I O N}

In this study, two sets of data were brought together from the literature to generate a spatiotemporal model of spinal MN activation during the locomotor step cycle. Several interesting aspects of the functional anatomy of the spinal cord and the migration of $\mathrm{MN}$ activity during locomotion emerged. Although some of these were known in vague terms or could have been deduced from the literature, the model presents them in a precise form.

It is important to identify the assumptions and limitations in our analysis. First, the anatomical study on which the work depends (VanderHorst and Holstege 1997) did not distinguish between alpha and gamma MNs, so the number of alpha MNs in a given pool is probably overestimated. We assume that the same bias occurred in all the MN pools, and so although the absolute numbers of alpha MNs is overestimated, the data provides an accurate picture of relative numbers of alpha MNs across pools.

Second, by making the number of active MNs in the spinal cord proportional to the amplitude of the EMG profile of a muscle, we tacitly assumed that EMG level is linearly related to net MUAP rate (and by extension to the net firing rate of corresponding spinal MNs). This is an important issue because if the relationship were highly nonlinear, the MN activity shown in the model could be significantly distorted. In an early human study, MU firing rates were found to increase with muscle force over the whole physiological range (Bigland and Lippold 1954). In separate studies, isometric muscle force was shown to be fairly linearly related to EMG (Basmajian and De Luca 1985). Taking these results together, it is safe to infer that MU firing rates increase with increasing EMG, but no firm conclusions can be made regarding the linearity of the relationship. In chronic recordings from single MNs during treadmill locomotion in cats, Hoffer et al. (1987b) found that MN firing rates in most muscles were linearly related to the corresponding mass EMG, at least over the range of gait velocities studied.

The relationship between EMG and net MN firing rate has also been analyzed in two theoretical studies. In the first (Person and Libkind 1970), EMG was modeled as the sum of triangular MUAP waveforms. The integrated EMG was roughly proportional to the square root of net MUAP rate when MUAPs were assumed to be asynchronous. The relationship became more linear with increasing synchronization. Rate modulation of MUAPs was neglected in this study, but in a more recent simulation using large numbers of realistic MUAP waveforms derived from single unit EMG recordings (Day and Hulliger 2001), this and other more physiological activation patterns were modeled. In most cases, EMG was more linearly related to the number of MUAPs than in the Person study, e.g., in the range $0-50 \% \mathrm{MU}$ recruitment, corresponding to medium-speed walking (Walmsley et al. 1978), mean EMG in- creased fairly linearly with the net MU firing rate (deviations from linearity $<10 \%$ ). Taking all this evidence together, the assumption of a linear relationship between EMG and net MUAP rate, although it is a simplification, is unlikely to produce major distortions in the estimates of net activity of MNs in the simulation.

Third, our EMG profiles were normalized so the assumption implicit in Figs. 4 and $5 A$ was that MN recruitment reaches the same maximal level in all muscles. This is also an oversimplification, and so we added two simulations in which the relative recruitment in flexors and extensors was varied. These parametric variations showed that although some of the subtleties of the migration of activity were either attenuated or exaggerated, overall the main features of spatiotemporal migration of activity were present in all the tests. The modeling would of course be better focused if it were possible to record from a large number of muscles in the same animal with similarly placed electrodes and identical signal processing.

Finally, some MN pools were not included in our analysis, either because EMG profiles were unavailable or because the anatomical data did not include them in enough detail. Generally these MN pools were much smaller than those we did include, the only notable exceptions being the large paravertebral muscles. Further data would be needed to fill in these gaps. However, we do not think that their inclusion would change our basic conclusions: first, the paravertebral muscles and their MN pools are distributed evenly over the whole lumbosacral region, so their activation profiles would add uniformly to those of the other pools and not significantly change the rostrocaudal migration of the center of activation. Second, back muscles are in any case not very active electromyographically during medium-paced locomotion at speeds less than $0.8 \mathrm{~m} / \mathrm{s}$ in the cat (Zomlefer et al. 1984).

Regarding our results, the first remarkable feature that emerges is the imbalance between the numbers of flexor and extensor MNs in the cat spinal cord. This leads to an imbalance in net MN activity during swing and stance phases, with a dominant seat of activity at a surprisingly caudal location (levels 80-85). If anything, our simplifying assumption that all MN pools reach the same maximal recruitment level underestimated the imbalance, as flexors such as TA and EDL are not strongly activated during swing phase compared with their activity in powerful voluntary withdrawal movements (Prochazka 1986), whereas MN recruitment in soleus, for example, may be virtually complete in locomotion (Walmsley et al. 1978). The actual recruitment balance during medium-paced locomotion is therefore probably most closely represented in Fig. $5 C$, where it is assumed that extensors are recruited to a maximum of $40 \%$ and flexors to a maximum of $15 \%$.

The preponderance of extensor MNs in both numbers and activity has a bearing on ideas about the structure of the locomotor CPG. Although Graham Brown probably did not mean his term "half-center" to be taken literally, the term does imply an equal weighting of flexor and extensor subdivisions (Brown 1911). The familiar schematic of the CPG, a circle bisected into flexor and extensor halves, continues this tradition. Our model does not of course give any information about the distributions of interneurons, of which the CPG may be largely composed (Tresch and Kiehn 1999), but at least at the MN level, it puts a different slant on flexor-extensor weighting. 
Note that it has been suggested that MNs are in fact integral elements of CPGs (Marder 1991; O’Donovan et al. 1998). Furthermore, recent studies have shown that interneurons and MNs in the same spinal segment share the same phase of activity in the step cycle (Tresch and Kiehn 1999), suggesting that the foci of MN activity in our model may also describe the activity of many associated interneurons but of course not necessarily all. Finally one should remember that $\mathrm{MN}$ firing rate profiles do not necessarily correspond completely to the time course of their locomotor drive potentials, e.g., some MNs may depolarize in two phases of the locomotor cycle but only fire action potentials in one phase (Perret and Cabelguen 1980).

The next feature to emerge is the rostrocaudal oscillation of foci of activity during the step cycle. As MN pools innervating muscles normally thought of as flexors (including BFp and ST) are distributed throughout the length of the lumbosacral enlargement, the alternation between a caudal focus of $\mathrm{MN}$ activity in the stance phase and a rostral focus in late swing was not a given. Our first impressions from the movie were of a wave of activation propagating smoothly back and forth along the spinal cord. Initially we felt that this was supported by the smoothness of the trajectories of the centers of activation we published in abstract form (Yakovenko et al. 2000). Propagation of activity along the neuraxis has been implicated in the orderly sequencing of activation of segmented musculature in different species (Orlovsky et al. 1999). However, the rostrocaudal transitions shown in Figs. 4 and 5 are in fact quite abrupt, so if smooth propagation from one set of neurons to the next is involved, it is rapid. Our model seems more consistent with the idea of abrupt switching between three groups of MNs: extensor MNs active during late swing and most of stance, retractor MNs being active in late stance and early swing and ankle and hip flexor MNs being active in swing only.

In the intact animal, stance-swing and swing-stance transitions in normal over-ground locomotion are under sensory control: when certain sensory conditions are met, phase switching is triggered (Prochazka 1993). It will therefore be interesting to compare the present results with those obtained when sensory input is absent (e.g., in fictive locomotion). In RESULTS, we pointed out a possible link between MN distribution and biomechanical action: in the pendant limb, rostral MNs would evoke forward movements of the foot and caudal MNs would evoke backward movements of the foot. However, in real-life locomotion, movements are affected by weight bearing so the activity of some muscles no longer fits this simple dichotomy. The spatiotemporal migration of activity in the spinal cord may then differ significantly from that in unloaded movements such as air-stepping and paw shakes.

Regarding BFp, gracilis, SMp, ST, and FDL, these muscles extend the hip and flex the knee and toes (gracilis also has an adductor role). Their main EMG bursts occur when the foot lifts off at the end of the stance phase (Engberg and Lundberg 1969; Halbertsma 1983), though FDL may also exhibit some activity earlier in stance (Loeb 1993; O'Donovan et al. 1982). In mid-stance, the moment arms of the posterior portions of BFp, gracilis, SMp, and ST about the knee are larger than those about the hip (Goslow et al. 1973). But in late stance and early swing, if the knee becomes very extended, the moment arms about hip and knee become similar. The biomechanical action at foot lift-off is therefore a backward and upward movement of the foot and toes with respect to the hip, this motion being crucial for ground clearance. Thus BFp, gracilis, SMp, ST, and FDL complete the backward movement of the foot that develops in the stance phase and initiate the upward movement that is then maintained by flexor muscles during swing. Sherrington (1910) listed gracilis, BFp, and ST muscles as being engaged in the flexion reflex of decerebrate cats, yet in his Table 2 , he lists BFp, FDL, and SM as being inhibited along with pure extensors when the flexion reflex was re-applied during a rebound extensor contraction. During reflex stepping and standing, BFp, gracilis, and ST behaved as pure flexors while SM and FDL were classified as extensors. Short-latency (presumed group Ia) reflex connections measured between various hip and knee muscles in lightly anesthetized cats added to the uncertainty of classifying BFp, gracilis, and ST as flexors (Eccles and Lundberg 1958).

In view of these various anomalies, as well as the results of our spinal cord modeling, we suggest that in locomotion BFp, gracilis, SMp, ST, and FDL muscles be classified as retractors, their role being to complete the backward movement of the foot and toes with respect to the hip (i.e., to complete the extensor thrust of the stance phase) and then to lift the foot in preparation for forward swing. This is not to deny that they can act differently in other tasks: e.g., BFp is strongly activated during flexion phases of withdrawal movements of the limb (Prochazka 1986) and gracilis has a significant adductor role (Sherrington 1910). From the point of view of the spatiotemporal flow of activity in the spinal cord, the activation of the BFp, gracilis, SMp, and ST MN pools involves a small transient shift caudad from the focus of extensor activity in the stance phase. Activity then switches rapidly to the rostral flexor centers.

Regarding the hypothesis of discrete "unit" oscillators controlling hip, knee, and ankle (Grillner and Wallen 1985; Kiehn and Kjaerulff 1998), Fig. 1 shows the actual anatomical positions of the three sets of MN pools that would be involved. If such unit oscillators exist and MNs are part of them (Marder 1991; O'Donovan et al. 1998), they are evidently widely spaced rostrocaudally, and so they would require long and overlapping propriospinal connections. For example, if we equate the centers of the unit oscillators with the centers of mass of their respective MN pools, the two components of the hip oscillator would be separated by 61 levels $(17.5 \mathrm{~mm}$ in an average-sized spinal cord), those of the knee oscillator by 58 levels $(16.7 \mathrm{~mm})$ and those of the ankle oscillator by only 20 levels (approximately $5.6 \mathrm{~mm}$ ). The rostrocaudal order of the knee flexor and extensor half-centers would be the reverse of that of the hip and ankle half-centers.

The approach introduced in this paper opens up a variety of interesting possibilities. Spatiotemporal patterns of spinal cord activation could be modeled and compared in various preparations (e.g., normal, decerebrate and spinal locomotion), in different types of cyclical movement (e.g., paw-shake response and scratching), and using different representations of $\mathrm{MN}$ activity e.g., intracellular recording. Models of this type could be extremely useful if and when spinal cord neuroprostheses are developed for people with spinal cord injury. The locomotor system of the cat spinal cord is not the only pattern generator amenable to this form of analysis. Any system in which the CNS distribution of neurons and the activity profiles of their target organs in the periphery are reasonably well 
characterized could be analyzed in this way. The dynamic models and the spinal cord movie described in this paper may be obtained in digital form from the authors.

This work was supported by grants from the Canadian Institutes of Health Research, the Alberta Paraplegic Foundation, and the Alberta Heritage Foundation for Medical Research.

\section{REFERENCES}

Abraham LD, Marks WB, AND Loeb GE. The distal hindlimb musculature of the cat. Exp Brain Res 58: 594-603, 1985.

Andersson O, Forssberg H, Grillner S, ANd Lindquist M. Phasic gain control of the transmission in cutaneous reflex pathways to motoneurones during "fictive" locomotion. Brain Res 149: 503-507, 1978.

ARMSTRONG DM AND MARPLE-HoRvat DE. Role of the cerebellum and motor cortex in the regulation of visually controlled locomotion. Can J Physiol Pharmacol 74: 443-455, 1996.

Arshavsky YI, Deliagina TG, and ORlovsky GN. Pattern generation. Curr Opin Neurobiol 7: 781-789, 1997.

BasmaJian JV and De LuCA CJ. EMG signal amplitude and force. In: Muscles Alive. Their Functions Revealed by Electromyography. Baltimore, MD: Williams and Wilkins, 1985, p. 187-222.

Belanger M, Drew T, Provencher J, and Rossignol S. A comparison of treadmill locomotion in adult cats before and after spinal transection. $\mathrm{J} \mathrm{Neu}$ rophysiol 76: 471-491, 1996.

BELANGER M, DRew T, AND Rossignol S. Spinal locomotion: a comparison of the kinematics and the electromyographic activity in the same animal before and after spinalization. Acta Biol Hung 39: 151-154, 1988.

BIGLAND B AND LIPPOLD O. The relation between force, velocity and integrated electrical activity. Motor unit activity in the voluntary contraction of human muscle. J Physiol (Lond) 125: 322-335, 1954.

Brown AG. Organization in the Spinal Cord: The Anatomy and Physiology of Identified Neurons. Berlin: Springer, 1981.

BRown TG. The intrinsic factors in the act of progression in the mammal. Proc Roy Soc Lond B Biol Sci 84: 308-319, 1911.

BuFORD JA AND SMITH JL. Adaptive control for backward quadrupedal walking. II. Hindlimb muscle synergies. J Neurophysiol 64: 756-766, 1990.

Carlson H, Halbertsma J, and Zomlefer M. Control of the trunk during walking in the cat. Acta Physiol Scand 105: 251-253, 1979.

Carrasco DI AND English AW. Mechanical actions of compartments of the cat hamstring muscle, biceps femoris. Prog Brain Res 123: 397-403, 1999.

CARRIER L, BRUSTEIN E, AND Rossignol S. Locomotion of the hindlimbs after neurectomy of ankle flexors in intact and spinal cats: model for the study of locomotor plasticity. J Neurophysiol 77: 1979-1993, 1997.

CAZAlets JR, Borde M, AND Clarac F. Localization and organization of the central pattern generator for hindlimb locomotion in newborn rat. J Neurosci 15: 4943-4951, 1995.

Chanaud CM, Pratt CA, ANd Loeb GE. Functionally complex muscles of the cat hindlimb. V. The roles of histochemical fiber-type regionalization and mechanical heterogeneity in differential muscle activation. Exp Brain Res 85: 300-313, 1991.

DAY SJ AND HUlliger M. Experimental simulation of cat electromyogram: evidence for algebraic summation of motor unit action potentials. J Neurophysiol 86: 2144-2158, 2001.

Deliagina TG, ORLOVSKY GN, AND PAVLOVA GA. The capacity for generation of rhythmic oscillations is distributed in the lumbosacral spinal cord of the cat. Exp Brain Res 53: 81-90, 1983.

Duysens J AND LOEB GE. Modulation of ipsi- and contralateral reflex responses in unrestrained walking cats. J Neurophysiol 44: 1024-1037, 1980.

ECCLES RM AND LUNDBERG A. Integrative pattern of Ia synaptic actions on motoneurones of hip and knee muscles. J Physiol (Lond) 144: 271-298, 1958.

Engberg I. Reflexes to foot muscles in the cat. Acta Physiol Scand Suppl 62: $1-64,1964$.

ENGBERG I AND LUNDBERG A. An electromyographic analysis of muscular activity in the hindlimb of the cat during unrestrained locomotion. Acta Physiol Scand 75: 614-630, 1969.

Fleshman JW, Lev-Tov A, AND BuRKe RE. Peripheral and central control of flexor digitorum longus and flexor hallucis longus motoneurons: the synaptic basis of functional diversity. Exp Brain Res 54: 133-149, 1984.

ForsSBERG H. On integrative motor functions in the cat's spinal cord. Acta Physiol Scand Suppl 474: 1-56, 1979.
Forssberg H, Grillner S, and Halbertsma J. The locomotion of the low spinal cat. I. Coordination within a hindlimb. Acta Physiol Scand 108: 269-281, 1980.

Goslow GE JR, Reinking RM, And Stuart DG. The cat step cycle: hind limb joint angles and muscle lengths during unrestrained locomotion. J Morphol 141: 1-41, 1973.

GRILLNER S. Locomotion in vertebrates: central mechanisms and reflex interaction. Physiol Rev 55: 247-304, 1975.

Grillner S, PARKER D, AND El MANIRA A. Vertebrate locomotion-a lamprey perspective. Ann NY Acad Sci 860: 1-18, 1998.

GRILlNeR S AND Rossignol S. On the initiation of the swing phase of locomotion in chronic spinal cats. Brain Res 146: 269-277, 1978.

GRILLNER S AND WALlen P. Central pattern generators for locomotion, with special reference to vertebrates. Аnпu Rev Neurosci 8: 233-261, 1985.

GRILLNER S AND ZANGGER P. How detailed is the central pattern generation for locomotion? Brain Res 88: 367-371, 1975.

GRILLNER S AND ZANGGeR P. On the central generation of locomotion in the low spinal cat. Exp Brain Res 34: 241-261, 1979.

Gritsenko V, Mushahwar V, and Prochazka A. Adaptive changes in locomotor control after partial denervation of triceps surae muscles. J Physiol (Lond) 533: 299-311, 2001.

HALBERTSMA JM. The stride cycle of the cat: the modelling of locomotion by computerized analysis of automatic recordings. Acta Physiol Scand Suppl 521: 1-75, 1983.

Hochman S, JORDAN LM, AND MACDONALD JF. $N$-methyl-D-aspartate receptor-mediated voltage oscillations in neurons surrounding the central canal in slices of rat spinal cord. J Neurophysiol 72: 565-577, 1994.

Hoffer Ja, Loeb Ge, Sugano N, Marks WB, O'Donovan MJ, and Pratt CA. Cat hindlimb motoneurons during locomotion. III. Functional segregation in sartorius. $J$ Neurophysiol 57: 554-562, 1987a.

Hoffer Ja, Sugano N, Loeb GE, Marks WB, O’Donovan MJ, and Pratt CA. Cat hindlimb motoneurons during locomotion. II. Normal activity patterns. J Neurophysiol 57: 530-553, 1987b.

JANKOWSKA E. Interneuronal relay in spinal pathways from proprioceptors. Prog Neurobiol 38: 335-378, 1992.

Kiehn O, Harris-Warrick RM, Jordan LM, Hultborn H, and Kudo N (Editors). Neuronal mechanisms for generating locomotor activity. Ann NY Acad Sci 860: 1-573, 1998

KieHN O AND KJAERUlfF O. Distribution of central pattern generators for rhythmic motor outputs in the spinal cord of limbed vertebrates. Ann NY Acad Sci 860: 110-129, 1998.

KREMER E AND LEV-Tov A. Localization of the spinal network associated with generation of hindlimb locomotion in the neonatal rat and organization of its transverse coupling system. J Neurophysiol 77: 1155-1170, 1997.

Lev-Tov A AND O'DonOvan MJ. Calcium imaging of motoneuron activity in the en-bloc spinal cord preparation of the neonatal rat. J Neurophysiol 74: $1324-1334,1995$.

LOEB GE. The distal hindlimb musculature of the cat: interanimal variability of locomotor activity and cutaneous reflexes. Exp Brain Res 96: 125-140, 1993.

LOEB GE AND HofFer JA. Activity of spindle afferents from cat anterior thigh muscles. II. Effects of fusimotor blockade. J Neurophysiol 54: 565-577, 1985.

Loeb GE, Hoffer JA, and Pratt CA. Activity of spindle afferents from cat anterior thigh muscles. I. Identification and patterns during normal locomotion. J Neurophysiol 54: 549-564, 1985.

MARCouX J AND Rossignol S. Initiating or blocking locomotion in spinal cats by applying noradrenergic drugs to restricted lumbar spinal segments. J Neurosci 20: 8577-8585, 2000.

MARDER E. Modifiability of pattern generator. Curr Opin Neurobiol 1: 571576, 1991.

MetTrie De La JO. L'Homme-Machine (Man A Machine; And, Man A Plant) [English translation: Watson RA (1994)]. New York: Gryphon Editions, 1745 .

Mushahwar VK, Collins DF, and Prochazka A. Spinal cord microstimulation generates functional limb movements in chronically implanted cats. Exp Neurol 163: 422-429, 2000.

MushaHWAR VK AND Horch KW. Selective activation and graded recruitment of functional muscle groups through spinal cord stimulation. Ann NY Acad Sci 860: 531-535, 1998.

O'Donovan MJ, Pinter MJ, Dum RP, AND Burke RE. Actions of FDL and FHL muscles in intact cats: functional dissociation between anatomical synergists. J Neurophysiol 47: 1126-1143, 1982. 
O'Donovan MJ, Wenner P, Chub N, TABaK J, And Rinzel J. Mechanisms of spontaneous activity in the developing spinal cord and their relevance to locomotion. Ann NY Acad Sci 860: 130-141, 1998.

Orlovsky GN, Deliagina TG, and Grillner S. Neuronal Control of Locomotion. Oxford, UK: Oxford Univ. Press, 1999.

PEARSON KG. Proprioceptive regulation of locomotion. Curr Opin Neurobiol 5: 786-791, 1995.

PerRet C AND CABELGUEN JM. Main characteristics of the hindlimb locomotor cycle in the decorticate cat with special reference to bifunctional muscles. Brain Res 187: 333-352, 1980.

PERSON RS AND LIBKIND MS. Simulation of electromyograms showing interference patterns. Electroencephalogr Clin Neurophysiol 28: 625-632, 1970.

PhiLIPPSON M. L'autonomie et la centralisation dans le système nerveux des animaux. Trav Lab Inst Solvay 7: 1-208, 1905.

Pratt CA, Buford JA, And Smith JL. Adaptive control for backward quadrupedal walking. V. Mutable activation of bifunctional thigh muscles. J Neurophysiol 75: 832-842, 1996.

Pratt CA, Chanaud CM, AND Loeb GE. Functionally complex muscles of the cat hindlimb. IV. Intramuscular distribution of movement command signals and cutaneous reflexes in broad, bifunctional thigh muscles. Exp Brain Res 85: 281-299, 1991.

Prochazka A. Proprioception during voluntary movement. Can J Physiol Pharmacol 64: 499-504, 1986.

PRochazKa A. Comparison of natural and artificial control of movement. IEEE Trans Rehab Eng 1: 7-16, 1993.

ProchazKa A. Proprioceptive feedback and movement regulation. In: Handbook of Physiology. Exercise: Regulation and Integration of Multiple Systems. New York: Am. Physiol. Soc., 1996, p. 89-127.

ProchaZKA A AND Gorassini M. Ensemble firing of muscle afferents recorded during normal locomotion in cats. J Physiol (Lond) 507: 293-304, 1998.

Prochazka A, Trend P, Hulliger M, and Vincent S. Ensemble proprioceptive activity in the cat step cycle: towards a representative look-up chart. Prog Brain Res 80: 61-74, 1989.

Prochazka A, Westerman RA, and Ziccone SP. Discharges of single hindlimb afferents in the freely moving cat. J Neurophysiol 39: 1090-1104, 1976.

Rasmussen S, Chan AK, AND Goslow GE JR. The cat step cycle: electromyographic patterns for hindlimb muscles during posture and unrestrained locomotion. J Morphol 155: 253-269, 1978.

Rho MJ, CABAna T, AND Drew T. Organization of the projections from the pericruciate cortex to the pontomedullary reticular formation of the cat: a quantitative retrograde tracing study. J Comp Neurol 388: 228-249, 1997.
Romanes GJ. The motor cell columns of the lumbo-sacral spinal cord of the cat. J Comp Neurol 94: 313-358, 1951.

Rossignol S, BARBEAU H, AND Julien C. Locomotion of the adult chronic spinal cat and its modification by monoaminergic agonists and antagonists. In: Development and Plasticity of the Mammalian Spinal Cord, Vol. III, edited by Goldberger ME, Gorio A, and Murray M. Padova: Liviana, 1986, p. 323-345.

SHARRARD WJW. The distribution of the permanent paralysis in the lower limb in poliomyelitis. J Bone Joint Surg 37: 540-558, 1955.

SHERRINGTON CS. Notes on the arrangement of some motor fibres in the lumbo-sacral plexus. J Physiol (Lond) 13: 621-772, 1892.

SHERRINGTON CS. Flexion-reflex of the limb, crossed extension-reflex, and reflex stepping and standing. J Physiol (Lond) 40: 28-121, 1910.

Smith JL, CARLSON-KuHTA P, AND TRANK TV. Forms of forward quadrupedal locomotion. III. A comparison of posture, hindlimb kinematics, and motor patterns for downslope and level walking. J Neurophysiol 79: 1702-1716, 1998.

Trank TV AND SMith JL. Adaptive control of backward quadrupedal walking. VI. Metatarsophageal joint dynamics and motor patterns of digit muscles. J Neurophysiol 75: 678-694, 1996.

TREnd P. Gain Control in Proprioceptive Pathways (PhD thesis). London, UK: University of London, 1987.

Tresch MC AND KIEHN O. Coding of locomotor phase in populations of neurons in rostral and caudal segments of the neonatal rat lumbar spinal cord. J Neurophysiol 82: 3563-3574, 1999.

VAnderHorst VGJM And Holstege G. Organization of lumbosacral motoneuronal cell groups innervating hindlimb, pelvic floor, and axial muscles in the cat. J Comp Neurol 382: 46-76, 1997.

WALMSley B, Hodgson JA, AND Burke RE. Forces produced by medial gastrocnemius and soleus muscles during locomotion in freely moving cats. J Neurophysiol 41: 1203-1216, 1978.

Wand P, Prochazka A, AND Sontag K. Neuromuscular responses to gait perturbations in freely moving cats. Exp Brain Res 38: 109-114, 1980.

Yakovenko S, Mushahwar VK, Vanderhorst V, Holstege G, and PROCHAZKA A. Locus of activation of spinal motoneuron pools in the cat step cycle. J Physiol (Lond) 525P: 42P, 2000.

Zhang W, Pombal MA, el Manira A, and Grillner S. Rostrocaudal distribution of 5-HT innervation in the lamprey spinal cord and differential effects of 5-HT on fictive locomotion. J Comp Neurol 374: 278-290, 1996.

Zomlefer MR, Provencher J, Blanchette G, and Rossignol S. Electromyographic study of lumbar back muscles during locomotion in acute high decerebrate and in low spinal cats. Brain Res 290: 249-260, 1984. 